\title{
Commentary for the Elderly in the Pandemic Era
}

\author{
Asif Khattak $^{\mathrm{a}}$ Badar Kanwar $^{\mathrm{a}}$ Consolato Sergi ${ }^{\mathrm{b}}$ Chul Joong Lee ${ }^{\mathrm{c}}$ \\ Jenny Balentine ${ }^{a}$ Jong-Hoon Lee ${ }^{d}$ Jungwuk Park ${ }^{e}$ So Jeong Lee ${ }^{f}$ \\ Su-Hee $\mathrm{Choi}^{\mathrm{g}}$
}

${ }^{a}$ Department of Pulmonary Critical Care Medicine, Hunt Regional Hospital, Greenville, TX, USA; ${ }^{b}$ Department of Laboratory Medicine and Pathology, University of Alberta, Edmonton, AB, Canada; ' Zein Pain Clinic Seongbuk, Seoul, Republic of Korea; ' Science and Research Center, Seoul National University College of Medicine, Seoul, Republic of Korea; ${ }^{e}$ Research Center of Integrative Functional Medicine, Department of Neurosurgery, Chungdam Hospital, Seoul, Republic of Korea; ${ }^{f}$ Department of BioSciences, Wiess School of Natural Sciences, Rice University, Houston, TX, USA; ${ }^{9}$ Department of Obstetrics and Gynaecology, Seoul National University Hospital, Seoul, Republic of Korea

In Korea, research into geriatric medicines started the Seoul Longitudinal Study on Aging from 1996 to 1999 [1] at the WHO Collaborating Center of Physical Culture and Aging Research for Health Promotion, Medical Research Center, Seoul National University, Seoul, Republic of Korea. The Seoul Longitudinal Study studied subjects (centenarians) older than 95 years of age [2]. Since 2001, various members of the Faculties of Medicine, Nutrition, Psychology, Family and Environmental Medicine, Demography, and Anthropology have participated in the nation-wide Korean Centenarian Study.

Some places in the world like Okinawa, Japan, and Sardinia, Italy, have an unusual number of individuals older than 100 years. There is a longevity village, Sunchang, in Korea. Centenarians living in mountainous regions have better health than those living near the sea. Similar findings have been noted in Sardinia, Italy. Habitat might influence gender differences in longevity, mainly via the influence of diet and physical activity [3].

One of the Centenarian Study researchers, S.C. Park, visited Sorok Island, where Hansen's disease (HD) pa-

karger@karger.com www.karger.com/dee

Karger"
(C) 2021 The Author(s)

Published by S. Karger AG, Basel

This is an Open Access article licensed under the Creative Common Attribution-NonCommercial-4.0 International License (CC BY-NC) (http://www.karger.com/Services/OpenAccessLicense), applicable to the online version of the article only. Usage and distribution for commercial purposes requires written permission. tients live, and found that the population had a long life expectancy [4-7]. He hypothesized that one of the reasons for this long life expectancy is that dapsone, a medicine for $\mathrm{HD}$, acts as an antioxidant. This investigation into whether dapsone contributes to longevity is ongoing.

J.L., who was conducting a study on the elderly diagnosed with mild cognitive impairment in 2008, began researching Alzheimer's disease and Parkinson's disease [8]. He created the Seoul cohort to study the action of the sulfone group of dapsone (under the assumption that this drug would expand cerebrovascular blood vessels). In December 2020, the study confirmed the action of dapsone on the neuroinflammasome $[9,10]$. In addition to the research into Alzheimer's disease, the findings of the HD studies are currently being clinically implemented to treat COVID-19 patients for acute respiratory distress syndrome (ARDS). Dapsone is also used to treat sequelae.

Dapsone binds noncovalently to the minor groove of DNA $[11,12]$. Until now, scientists regarded dapsone as an adjuvant [13], alternative [14], augmentation [15, 16], or active ingredient $[6,17]$ for improved outcomes for
Correspondence to:

Jong-Hoon Lee, science@ research.re.kr 


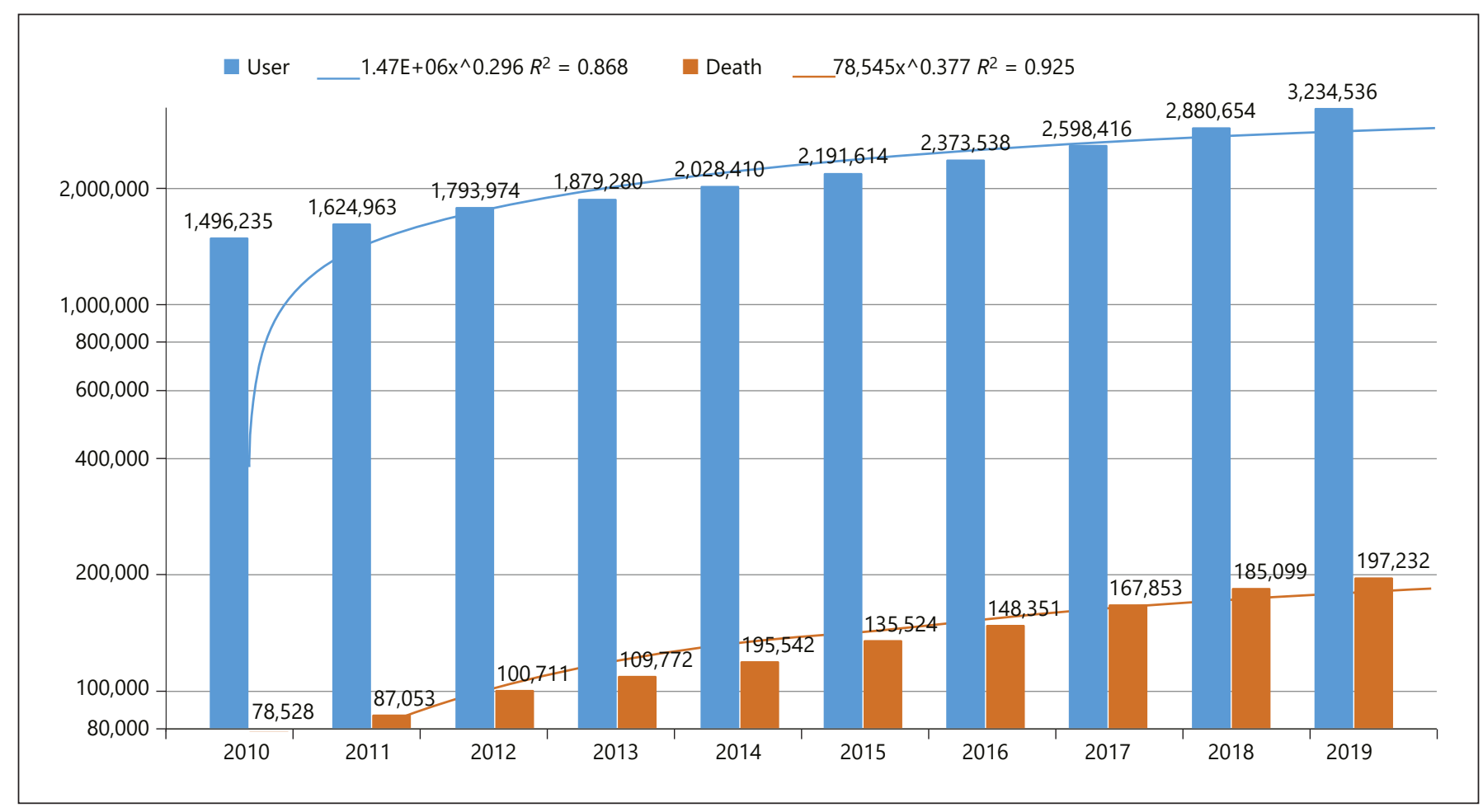

Fig. 1. Graph of total dementia drug prescriptions and deaths of Alzheimer's disease patients in Korea. The trend line is a power series. Around the revision of the Dementia Act in 2018, the prescription of dementia drugs and deaths increased together. Psychiatric drugs: aripiprazole, olanzapine, risperidone, quetiapine, hal- operidol, selective serotonin reuptake inhibitors, and carbamazepine. Dementia symptom-improving drugs: donepezil, galantamine, rivastigmine, and memantine. These data are from an entire database of the Korea Health Insurance Corporation. patients. Newly developed drugs are used to treat significant diseases, but dapsone improve treatment efficiency.

To date, dapsone is used as a treatment and preventive drug for mild cognitive impairment $[8,18]$, Alzheimer's disease [10], Parkinson's disease [19, 20], seizure [21], stroke $[9,22,23]$, and COVID-19 ARDS [11, 13].

We present the data on the increase and decrease in deaths and the psychiatric drugs administered with 4 types of dementia symptom-improving drugs from 2010 to 2019 (Fig. 1).

The medicines mainly administered to the elderly are closely related to health insurance policies. Of course, if the elderly die quickly, health insurance companies benefit. However, health insurance policies are operated to maintain the health of the elderly. If dapsone were used for the early symptoms of cognitive impairment or stroke, many earlier deaths could be prevented. Recently, the team of B.K. at the Hunt Regional Hospital reported a study that drastically reduced mortality by administering dapsone to patients with COVID-19 ARDS in the intensive care unit [11, 13]. J.M.'s research team, who studied brain autopsy findings of COVID-19 patients, supported the findings of B.K.'s team by demonstrating the impact of SARS-CoV-2 infection in the medulla oblongata's pre-Bötzinger complex [24].

\section{Methods}

We connected to the Korea Health Insurance Corporation's medical records database and archived it from 2010 to 2019. With the ICD-9 and ICD-10 codes, medical data on the correlation between Alzheimer's disease and dementia drugs were then analyzed for cohort correlational possibility.

List of ICD Codes and Diseases

F00, dementia in Alzheimer's disease (G30.-+); F01, vascular dementia, F02, dementia in other diseases classified elsewhere; F03, unspecified dementia; F04, organic amnesic syndrome, not induced by alcohol and other psychoactive substances; F05, delirium, not induced by alcohol and other psychoactive substances; F06, other mental disorders due to brain damage and dysfunction, and to physical disease; F07, personality and behavioral disorders 
due to brain disease, damage, and dysfunction; F09, unspecified organic or symptomatic mental disorder; G30, Alzheimer's disease.

\section{Dementia Drugs according to the Korea Drug Code of} Medicines

First-group dementia drugs are administered for symptomatic relief in Alzheimer's disease. Examples are: donepezil hydrochloride (148603ATB 148602ATD 148602ATB 148601ATD 148601ATB 643401ATD 643402ATD), rivastigmine (224501ACH 224503ACH 224504ACH 224505ACH 224506CPC 224507CPC 224508CPC), galantamine (385203ACR 385203ATR 385204ACR 385204ATR 385205ACR 385205ATR), and N-methyl-D-aspartate [NMDA] receptor antagonist (190031ALQ 190001ATB 190003ATD 190004ATB 190004ATD).

Second-group drugs are administered for psychological symptoms of Alzheimer's disease. Examplesare:haloperidol(167903ATB 167904ATB 167905ATB 167906ATB 167908ATB 167908ATB 168030BI), risperidone (224201ATB 224201ATD 224202ATB 224202ATD 224203ATB 224204ATB 224205BIJ 224206BIJ), quetiapine (378601ATB 378602ATB 378603ATB 378604ATB 378605ATB 378605ATR 378606ATR 378607ATR 378608ATR 378608ATR 378610ATB), olanzapine (204001ATB 204001ATD 204002ATB 204002ATD 204004ATB 204005ATB), aripiprazole (451501ATB 451501ATD 451502ATB 451502ATD 451503ATB 451504ATB 451505ATB 451506BIJ 451507BIJ), oxcarbazepine (206330ASS 206301ATB 206302ATB 206303ATB), fluvoxamine (162501ATB 162502ATB), escitalopram (474801ATB 474802ATB 474803ATB 474804ATB), trazodone (242901ACH 242901ATB 242902ATB 242903ATR), sertraline (227001ATB 227002ATB 227003ATB), and fluoxetine (161501ACH 161501ATB 161502ACH 161502ATB 161502ATD).

\section{Statement of Ethics}

The Bioethics Committee, a central institution designated by the Ministry of Health and Welfare, approved the observational study of patients ethically based on FDA guidelines following the World Medical Association Declaration of Helsinki (P01-20200722-006). We carried out all methods following relevant ethics guidelines and regulations.

\section{Conflict of Interest Statement}

The authors have no conflicts of interest to declare.

\section{Funding Sources}

There are no funding sources to declare.

\section{Author Contributions}

J.L. discussed with C.J.L., J.P., S.J.L., and S.-H.C., who studied Alzheimer's disease and stroke to write this paper. J.L. discussed SAR-CoV-2 treatment with A.K., B.K., C.S., and J.B. The contents and conclusions of this paper were reviewed by all authors. J.L. wrote the paper.

\section{References}

1 Yun YG. Seoul Longitudinal Study on Aging. In: Welfare $\mathrm{MoHa}$, editor. Seoul: Ministry of Health and Welfare; 1999. p. 42.

2 Kwak CS, Cho JH, Yon M, Park SC. Anthropometric index, dietary habits and nutrient intake of the oldest-old population aged 95 and over living in Seoul. Korean J Community Nutr. 2012;17(5):603-22.

3 Park S, Lee M, Kwon I, Kwak C, Yeo E. Environment and gender influences on the nutritional and health status of Korean centenarians. Asian J Gerontol Geriatr. 2008;3(2):7583.

4 Cho SC, Rhim JH, Son YH, Lee SJ, Park SC. Suppression of ROS generation by 4,4-diaminodiphenylsulfone in non-phagocytic human diploid fibroblasts. Exp Mol Med. 2010 Mar;42(3):223-32.

5 Cho SC, Park MC, Keam B, Choi JM, Cho Y, Hyun S, et al. DDS, 4,4'-diaminodiphenylsulfone, extends organismic lifespan. Proc Natl Acad Sci USA. 2010 Nov;107(45):19326-31.

6 Park SC, Lee J, Cho SC, Park MC, Cho YJ. Composition for control of aging and/or extension of life, containing dapsone as active ingredient. Google Patents; 2011.
7 Cho SC, Rhim JH, Choi HR, Son YH, Lee SJ, Song KY, et al. Protective effect of $4,4^{\prime}$-diaminodiphenylsulfone against paraquat-induced mouse lung injury. Exp Mol Med. 2011 Sep;43(9):525-37.

8 Lee JH, Choi SH, Lee CJ, Oh SS. Recovery of Dementia Syndrome following Treatment of Brain Inflammation. Dement Geriatr Cogn Disord Extra. 2020 Jan;10(1):1-12.

9 Lee Jh, Lee CJ, Park J, Lee SJ. The neuro-inflammasome in Alzheimer's disease and cerebral stroke. Dementia and Geriatric Cognitive Disorders EXTRA. 2021; under review.

10 Jong-hoon L, Chul Joong L, Jungwuk P, So Jeong L, Su-Hee C, Sang-Suk O. The Preventive and Treatment of the Neuro-Inflammasome in Sorokdo National Hospital [under review]. Sci Rep. 2021.

11 Chakraborty A, Panda AK, Ghosh R, Biswas A. DNA minor groove binding of a well known anti-mycobacterial drug dapsone: A spectroscopic, viscometric and molecular docking study. Arch Biochem Biophys. 2019 Apr;665:107-13.
12 Lee JH, An HK, Sohn MG, Kivela P, Oh S. $4,4^{\prime}$-Diaminodiphenyl Sulfone (DDS) as an Inflammasome Competitor. Int J Mol Sci. 2020 Aug;21(17):5953.

13 Tirado-Sánchez A, Díaz-Molina V, PonceOlivera RM. Efficacy and safety of azathioprine and dapsone as an adjuvant in the treatment of bullous pemphigoid. Allergol Immunopathol (Madr). 2012 May-Jun;40(3):152-5.

14 Salehzadeh F, Jahangiri S, Mohammadi E. Dapsone as an alternative therapy in children with familial mediterranean Fever. Iran J Pediatr. 2012 Mar;22(1):23-7.

15 Tamarkin D, Friedman D, Eini M. Anti-infection augmentation foamable compositions and kit and uses thereof. Google Patents; 2007.

16 Kast RE. Erlotinib augmentation with dapsone for rash mitigation and increased anticancer effectiveness. Springerplus. 2015 Oct;4(1):638.

17 McGeer PL, Harada N, Kimura H, McGeer EG, Schulzer M. Dapsone and promin for the treatment of dementia. Google Patents; 1996. 
18 Yang N, Li L, Li Z, Ni C, Cao Y, Liu T, et al. Protective effect of dapsone on cognitive impairment induced by propofol involves hippocampal autophagy. Neurosci Lett. 2017 May;649:85-92.

19 Sulzer D, Antonini A, Leta V, Nordvig A, Smeyne RJ, Goldman JE, et al. COVID-19 and possible links with Parkinson's disease and parkinsonism: from bench to bedside. Parkinsons Dis. 2020;6(1).

20 Zhao K, Lim YJ, Liu Z, Long H, Sun Y, Hu JJ, et al. Parkinson's disease-related phosphorylation at Tyr39 rearranges $\alpha$-synuclein amy- loid fibril structure revealed by cryo-EM. Proc Natl Acad Sci USA. 2020 Aug;117(33):2030515.

21 Kast RE, Lefranc F, Karpel-Massler G, Halatsch ME. Why dapsone stops seizures and may stop neutrophils' delivery of VEGF to glioblastoma. $\mathrm{Br}$ J Neurosurg. 2012 Dec;26(6):813-7.

22 Nader-Kawachi J, Góngora-Rivera F, SantosZambrano J, Calzada P, Ríos C. Neuroprotective effect of dapsone in patients with acute ischemic stroke: a pilot study. Neurol Res. 2007 Apr;29(3):331-4.
23 Cruz-Cruz C, Kravzov-Jinich J, MartínezNúñez JM, Ríos-Castañeda C, Perez ME, Altagracia-Martínez M. Cost-utility analysis in acute ischemic stroke survivors treated with dapsone in a public hospital in Mexico City. J Pharm Health Serv Res. 2014;5(2):95102

24 Meinhardt J, Radke J, Dittmayer C, Franz J, Thomas C, Mothes R, et al. Olfactory transmucosal SARS-CoV-2 invasion as a port of central nervous system entry in individuals with COVID-19. Nat Neurosci. 2021;24:16875 . 